\title{
MIT ODER OHNE OHREN, MIT ODER OHNE SCHALL Theorie- und Begriffsarbeit in den Sound Studies
}

von MAREN HAFFKE

James A. Steintrager, Rey Chow (Hg.): Sound Objects, Durham, London (Duke University Press) 2019

Britta Herrmann, Lars Korten (Hg.): Diskurse des Sonalen, Berlin (Vorwerk 8) 2019

Kerstin Ergenzinger: Navigating Noise, hg. v. Nathanja van Dijk, Kerstin Ergenzinger, Christian Kassung, Sebastian Schwesinger, Berlin (Verlag der Buchhandlung Walther König) 2017

Es gehört zur Institutionalisierung von Fächern und Forschungsfeldern, dass diese sich über ihre Gegenstände vergewissern. Theorie- und Begriffsarbeit als Teil fachpolitischer Ortsbestimmungen finden derzeit im interdisziplinären Feld der Sound Studies statt, das laut Axel Volmar und Bettina Schlüter in eine Konsolidierungsphase eintritt. ${ }^{1}$ Die hier vorgestellten Sammelbände behandeln die Objekte und Terminologien, Epistemologien und Institutionen der Sound Studies, indem sie Medienverhältnisse zwischen Psychoakustik und Akustik, Information und Noise befragen. ${ }^{2}$ Sie adressieren damit eine innerhalb des Feldes geführte Debatte darüber, ob der Gegenstand der Sound Studies eher Kulturen des Hörens, mit Schall befasste Medien oder eine darüber hinausreichende Ordnung der Signale sein sollen bzw. wie sich diese unterschiedlichen Bereiche zueinander verhalten. Die Debatte ist eingetragen in ein Spannungsfeld klanglicher Theoriebildung, dessen Kartierung bislang zumeist anhand der Gegenüberstellung zweier Sammelbegriffe organisiert wird: Auditory Culture Studies - verstanden als eine den Visual Culture Studies vergleichbare Kulturwissenschaft vom Hören und Hörbaren - und Sound Studies - verstanden als eine technisch-materialistisch orientierte Disziplin mit stärkerer naturwissenschaftlicher Ausrichtung. ${ }^{3}$ Diese Gegenüberstellung dient in vielen Entwürfen zugleich als Aufriss zweier weiterer Spannungen innerhalb des Feldes: Es geht zum einen um eine Unterscheidung von Theorien der Repräsentation von materialistischen Theorien (worunter sowohl Medientheorien fallen als auch Theorien unmittelbarer materieller Effekte) und zum anderen um das Verhältnis epistemologischer Perspektiven zu solchen Theorien, die sich explizit als Ontologien entwerfen.

In verschiedenen jüngeren Arbeiten des Feldes werden diese Konstellationen derzeit problematisiert. Autor_innen wie Marie Thompson, Brian Kane und auch ich selbst kritisieren nicht nur den vermeintlichen Gegensatz zweier theoretischer Bezugssysteme entweder von Konzepten der Repräsentation oder von Konzepten der Materialität als falsche Opposition, sondern vor allem auch die Tendenz zur Ontologisierung dieser Opposition und die damit zum Teil mit-ontologisierte Verunsichtbarung bestimmter Fragen von Gender und race auf Seiten der materialistischen Theorien. ${ }^{4}$

Die drei vorgestellten Bände stellen Perspektiven auf die Fachgeschichte des Feldes vor, auf sein gegenwärtiges Verhältnis zu anderen Fachkulturen und auf inter- und transdisziplinäre theoretische Potenziale, 
indem sie eigene begriffliche Schwerpunkte setzen. In den Fokus genommen werden dabei spezifische Beziehungen: Verhältnisse der musikalischen Avantgarden zur French Theory im Band Sound Objects, Verhältnisse von Literaturgeschichte zur Klangforschung im Band Diskurse des Sonalen und Verhältnisse von bildender Kunst zur Medienphilosophie an den Schnittstellen von Sound-Theorie und Artistic Research im Band Navigating Noise.

Mit Sound Objects stellen die Herausgeber_innen, der Literaturwissenschaftler James A. Steintrager und die Kulturwissenschaftlerin Rey Chow, in Aussicht, Verhältnisse von Theorie und Klang in zweifacher Weise epistemologisch zu situieren: Zum einen geht es darum, mögliche theoretische Zugriffe auf jene Gegenstände zu entwickeln, die anhand des titelgebenden Konzepts der sound objects versammelt werden. Zum anderen soll die Frage nach der Rolle von Sound in der Geschichte von theory selbst gestellt werden. Mit ihrem Theoriebegriff rufen Steintrager und Chow vor allem die spezifische Rezeptionsgeschichte strukturalistischer und poststrukturalistischer Theorien im Kontext der nordamerikanischen akademischen Tradition auf. ${ }^{5}$ Die Konzepte und Fragestellungen der jeweiligen theoretischen Perspektivierungen werden dabei sowohl historisiert als auch auf ihre Anwendbarkeit und ihre analytischen Potenziale überprüft. Diese ohnehin ambitionierte Zielsetzung des Sammelbandes erhält weitere Komplexität durch die Wahl der konzeptuellen Rahmung, anhand derer die genannten epistemologischen Konstellationen entfaltet werden sollen: die besagte Terminologie der sound objects, die auf die Theorie und Praxis der Musique concrète des Komponisten Pierre Schaeffer verweist.

Steintragers und Chows Bezug auf Schaeffer ist zugleich naheliegend und kontraintuitiv: Sie stellen ihn als eigentliche Vaterfigur der Sound Studies vor. Das ist, wenn man so will, nicht völlig abwegig - so man denn wirklich im heterogenen Feld der Sound Studies einen patriarchalischen Gründungsmythos ins Werk setzen möchte (siehe «Sound Objects. An Introduction", S.1-19). ${ }^{6}$ Natürlich muss man Fachgeschichte nicht so schreiben. Steintragers und Chows Erklärung Schaeffers zum wortwörtlichen "patriarch» (S.14) der
Sound Studies bleibt daher überraschend (zumal ihre Zusammenarbeit mit einer gemeinsamen Herausgabe der Zeitschrift differences. A Journal of Feminist Cultural Studies begann). Überraschend ist auch die Argumentation, mit der sie begründet wird: So erkläre sich eine besondere diskursive Produktivität der Schaeffer'schen Theorietradition laut Steintrager und Chow dadurch, dass Schaeffers Schriften lange nicht in englischer Übersetzung vorlagen und die englischsprachige Akademie vor allem durch ihre Rezeption in der Arbeit Michel Chions erreichten. Der mutmaßliche Vater der Sound Studies autorisiert den Diskurs also in Abwesenheit.

Eine solche These muss nicht unbedingt zutreffend sein, um sich als produktiv zu erweisen. Die behauptete diskursbegründende Rolle von Schaeffers Konzepten für die Sound Studies scheint im Spiegel der Beiträge eher befragt als gestärkt zu werden. Dabei werden Momente der Übersetzung, der Übertragung und der Migration von Konzepten zwischen verschiedenen Disziplinen und akademischen Traditionen in den Blick gerückt, die relevante und zum Teil wenig erschlossene Perspektiven für die Sound Studies entwickeln. Anhand der titelgebenden sound objects wird so nicht nur thematisiert, wie das Klingende bei Schaeffer zum Objekt wird, sondern auch und gerade, wie Sound zum epistemischen Objekt der Sound Studies wird.

Der Begriff objet sonore verweist in Schaeffers Arbeit auf die spezifische Qualität einer durch technische Aufzeichnung und/oder durch eine Lenkung der Wahrnehmung von ihren indexikalischen Dimensionen getrennten Klanglichkeit, die als grundlegende Einheit für musikalische Operationen jenseits instrumenteller Tonerzeugung bestimmt wird. Sound object ist die bis heute gängigste englische Übersetzung von objet sonore. In seinem Beitrag «The Fluctuating Sound Object» verweist Brian Kane auf eine semantische Drift des Begriffes, die im Französischen von der Alltagsbedeutung als Klangkörper oder «klingendes Objekt zum Klingenden als Objekt führt und in der englischen Übersetzung eine weitere Verschiebung durch die Substantivierung vom Sound im Singular erfährt. Diese Bewegung stellt Kane in den Kontext gegenwärtiger Bezugnahmen auf 
Schaeffers Klassifizierung in Debatten innerhalb der Sound Studies, welche die Selbstbeschreibung des Feldes zwischen kulturwissenschaftlichen Anschlüssen und einer Proliferation materialistischer Ontologien des 'Vibrationalen ${ }^{7}$ betreffen.

In Kanes Beitrag wird Schaeffers begriffliche Systematik zum Ausgangspunkt für Fragen nach möglichen theoretischen Zugriffen auf die Materialität von Klang, die diese Ontologisierungstendenzen selbst zu adressieren erlauben, ohne dabei die spezifische diskursive Produktivität materialistischer Perspektiven preiszugeben. So zeigt seine Analyse anhand der Schaeffer-Rezeption zweier von ihm untersuchter akustischer Ontologien (von Christoph Cox und Francisco López, die beide über Sound Art schreiben), wie Materialität in den entsprechenden Entwürfen je konkret verortet wird. Kane weist insbesondere darauf hin, dass beide betrachteten Positionen das Schaeffer'sche Konzept vom Sound als Objekt explizit zurückweisen - zugunsten eines temporalen Verständnisses von Sound als Ereignis, das Prozesse der Subjektivierung und Objektivierung in den Blick rückt.

Diese im Negativbezug auf Schaeffer produktiv gemachte Problematisierung sowohl des Soundbegriffs als auch des Objektbegriffs findet in einem Band statt, der beide Termini mit vergleichsweise großem semantischem Spielraum behandelt. Während die Herausgeber_innen den Begriff der Theorie aufgrund seiner konzeptuellen Verbindungen zu Verhältnissen von Wahrheit und Sichtbarkeit hinsichtlich seiner Eignung für Sound-Diskurse vor dem Hintergrund einer bis zu Immanuel Kant zurückverfolgten Geschichte ästhetischer Theorie ausführlich diskutieren, bleiben die beiden Teilbegriffe des Titels seltsam unbestimmt. Neben den direkt mit Schaeffer beschäftigten Beiträgen von Michel Chion und John Dack wird die Rede vom sound object so zum Ausgangspunkt für sehr unterschiedlich situierte Perspektivierungen von Klang als Objekt zwischen sonorer Gegenständlichkeit und auditiver Verdinglichung: Während der Beitrag von Steintrager etwa musikalische Objektivität bei Adorno mit einem kurzen elliptischen Bezug auf Schaeffer behandelt, schreibt Jonathan Sterne über klangliche Warenförmigkeit als Teil der Fetischisierung von Soundtechnik in gegenwärtigen Praktiken der Produktion und Konsumption von Musik - gänzlich ohne Schaeffer-Verweis. ${ }^{8}$ Vor dem Hintergrund dieser heterogenen Tiefenbohrungen in den Beiträgen ist es schade, dass in der Einleitung kaum auf eine mögliche terminologische Binnendifferenzierung zwischen aurality, sound und noise eingegangen wird. Etablierte begriffliche Unterscheidungen in der jeweiligen Gewichtung von Wahrnehmung, Schallereignis und Signal werden so zugunsten einer allgemeinen Gegenüberstellung von Hör-Wissen und visueller Theoriebildung zurückgestellt.

Eine solche terminologische Binnendifferenzierung soundtheoretischer Diskursebenen stellt der von den Germanist_innen Britta Herrmann und Lars Korten herausgegebene Band Diskurse des Sonalen vor. Der Band behandelt eine begriffliche und theoretische Arbeit am Klang anhand der Frage nach der Rolle von (Klanglichkeit) für die Poetik- und Literaturgeschichte. So plädiert Herrmann in ihrer Einleitung dafür, «Literaturforschung nicht nur medienkulturwissenschaftlich zu betreiben, sondern sie zugleich an die wachsenden Sound Studies anzuschließen" (S.9). Als zweiter Band der Reihe Klang - Kunst - Kultur knüpft das Buch an terminologische Vorarbeiten des 2015 von Herrmann herausgegebenen Bandes Dichtung für die Ohren an, in dem die beiden Begriffe des Tonalen und des Auralen zur Differenzierung einer «sonalen` Dimension literarischer Werke vorgeschlagen werden. Ersterer verweise auf die Ebene der Produktionsästhetik und Zweiterer auf die Ebene der Rezeptionsästhetik von Texten. Mit der begrifflichen Trias von sonal, tonal und aural interveniert Herrmann in die etablierte Dichotomie von Oralität und Literalität und stellt zugleich eine Ergänzung zum Begriff des Visuellen vor.

Nicht ersetzen, sondern anreichern soll die Neuprägung bestehende terminologische Traditionen innerhalb der Sound Studies, die laut Herrmann gerade in ihrer Interdisziplinarität und Vielstimmigkeit produktiv 
werden. Außer auf das Konzept von Sound, das sie dem kulturwissenschaftlichen Zweig zuweist, beruft sie sich hier auch auf den zuletzt unter anderem von Wolfgang Ernst popularisierten Begriff des Sonischen, der innerhalb der Tradition der deutschsprachigen materialistischen Medienarchäologie und ihrer spezifischen Rezeption der Informationstheorie für ein Programm der technischen Signalanalyse als dezidierte Abwendung von kulturwissenschaftlichen Fragestellungen steht. Während beide genannten Begriffe (Sound und das Sonische) Schallereignisse zum Gegenstand haben, soll der Begriff des Sonalen die mediatisierte Tonalität von Texten erfassen und damit eine mediale Ebene auraler und tonaler Erfahrungen adressieren, die nicht auf akustische Energie verweist, sondern durch phonetische Zeichen der Schrift oder Bilder vermittelt wird.

Dieser Vorschlag erscheint überaus sinnvoll. Medienwissenschaftlich relevant ist vor allem die explizite Markierung psychoakustischer Phänomene als medial: So konturiert die mit dem Sonalen bezeichnete klangliche Dimension von Texten eine Medialität von «Klang ohne Schall, die in den Sound Studies bisher nicht zuletzt im Anschluss an Friedrich Kittler als psychoakustische Simulation noch nicht verfügbarer akustischer Medien in den Blick genommen wurde - als Unmittelbarkeitseffekt auf Seiten liebevoll alphabetisierter Leser_innen des 19. Jahrhunderts. ${ }^{9}$ Mit dem Begriff des Sonalen werden textlich vermittelte Hörerlebnisse stattdessen als historisch je spezifisch situierte materielle Relationen in den Blick genommen, die Verhältnisse von Akustik und Alphabetisierung, Hörund Lesekulturen erst vermitteln.

Herrmann verkompliziert damit zugleich die McLuhan'sche These von einer Ablösung des Ohrs durch das Auge als Übergang vom Vortrag zur stillen Lektüre am Beginn der Moderne und verweist auf komplexe Verflechtungen von Lese- und Vorlesepraktiken, die über das 18. Jahrhundert hinaus zu beobachten seien und dabei an je gegebenes musikalisches und akustisches Wissen sowie an spezifische Dimensionen auraler Sozialität anschlössen. Der Band versammelt dazu Beiträge, die von der Literatur und Musik des 18. Jahrhunderts bis zu den Medien und der Klangkunst des 21. Jahrhunderts reichen und unter anderem den Sprachursprung bei Rousseau behandeln (Laure Spaltenstein), Anti-Vertonung in Christian Morgensterns "Fisches Nachtgesang" (Anne Holzmüller), Konzepte des Sonalen in Richard Wagners Tristan und Isolde (Bettina Schlüter) und die Geschichte des Sound-Writing (Marc Matter).

Dass mit dem Sonalen aus Sicht der Literaturwissenschaft die Medialität von «Klang ohne Schall` fokussiert wird, ist für die Sound Studies nicht zuletzt deshalb konstruktiv, weil deren Fachgeschichte - vor allem im deutschsprachigen akademischen Kontext - insbesondere Materialitätskonzepte produktiv gemacht hat, die umgekehrt 'Schall ohne Klang) als Gegenstand etablierten: vibrationale Ereignisse, akustische Energie und Zeitverhältnisse jenseits der menschlichen Wahrnehmung. Es ist diese Ebene, die der schon erwähnte Begriff des Sonischen konturiert. ${ }^{\mathbf{1 0}}$ Und es ist diese Ebene, die in diversen materialistischen Theorien Ontologisierungen zugeführt wird. Der dabei deutlich werdende posthumanistische Gestus betont die Eingrenzung (und zum Teil Abwertung) menschlicher Perzeption als Teilmenge einer darüber hinausreichenden Welt der Wellen und Pulse, die erst technisch verfügbar werden. Dies verweist auf die zentrale Rolle einer bis auf die 1970er Jahre zurückreichenden Rezeption der Informationstheorie in Theorien akustischer Medien sowie auf die wichtige Rolle materieller Medientheorien der 1980er und 1990er innerhalb der Epistemologie der Sound Studies. ${ }^{11}$

In dieser Tradition steht der Band Navigating Noise, der von der Kuratorin Nathanja van Dijk, der Künstlerin Kerstin Ergenzinger und den Kulturwissenschaftlern Christian Kassung und Sebastian Schwesinger herausgegeben wurde. Mit dem Noise ist ein zentrales Konzept der Sound Studies im Anschluss an die Informationstheorie aufgerufen. ${ }^{\mathbf{1 2}}$ Navigating Noise ist darüber hinaus der Name einer von Ergenzinger gemeinsam mit dem Physiker Thomas Laepple entwickelten 
Sound-Art-Installation, die im Zentrum einer 2015 in Berlin veranstalteten Konferenz stand. Dass die Dokumentation eines Kunstwerks zum Ausgangspunkt für darum versammelte Theoriebeiträge wird, verweist institutionell auf bestehende Anschlüsse soundtheoretischer Arbeit zu den Institutionen der bildenden Kunst sowie auf spezifische Verhältnisse von Artistic Research zu dem, was man lange mit leichter Herablassung als ¿Kunsthochschulphilosophie) bezeichnet hat. Diese Konstellation ist weder selbstverständlich noch trivial. Relevante und paradigmenbildende theoretische Arbeit im Bereich der Sound Studies, die über den Kunstkontext hinaus rezipiert wird, bezieht sich auf Contemporary Sound Art, die selbst wiederum innerhalb der Geschichte der Avantgarden zu verorten ist: von Theoretiker_innen wie Brandon LaBelle über den schon erwähnten Christoph Cox bis Douglas Kahn. ${ }^{13}$

Auch die Beiträge in Navigating Noise beziehen sich zum Teil direkt auf Ergenzingers Arbeit. In ihnen geht es um Strategien 'sonischen Denkens', die Philosophie und künstlerische Praxis verschränken und dabei zugleich Perspektiven auf naturwissenschaftliche und sozialwissenschaftliche Klangforschung entwickeln. Der NoiseBegriff wird dabei als Materialitätskonzept perspektiviert und zugleich in emphatischer Weise als Ressource der Potenzialität, des Dynamischen und des Unvorhersehbaren mobilisiert. Viele der Texte erheben einen Anspruch auf Gegenwartsdiagnostik und stellen ihre Theoriearbeit in den Kontext einer Suche nach adäquaten Beschreibungsformen für eine sich radikal verändernde technische Welt. Der Beitrag von Eleni Ikoniadou fragt so nach einer kommenden Ästhetik klanglichen Denkens, die der medialen Spezifik von Sound gerecht zu werden habe, und situiert ihre Suchbewegungen in einer Vielzahl philosophischer Referenzen - darunter diverse Verweise auf Positionen aus der Nähe des Spekulativen Realismus. Dass diese innerhalb der Sound Studies eine vergleichsweise starke Rezeption erfahren haben, lässt sich sowohl auf den institutionellen Anschluss des Feldes an die Kunstwelt erklären (wo ebenfalls eine starke Rezeptionsgeschichte vorliegt) als auch durch die diskursive Nähe der Spekulativen Realist_innen zu den innerhalb der Sound Studies epistemologisch einflussreichen materialistischen Medientheorien hinsichtlich von Fragen der Repräsentationskritik.

Während die Sound Studies es in der Tat schwer hatten, ihre Fragestellungen an semiotische Traditionen poststrukturalistischer Theoriebildung anzuschließen, erwiesen sich die explizit gegen Konzepte der Signifikation Stellung beziehenden Medientheorien der 1980er und 199oer Jahre im Zuge der Formierung des Faches als produktiv. Es waren nicht zuletzt medienmaterialistische Perspektiven, die Noise als rauschenden Überschuss der Schrift bestimmten und fachpolitische Reformulierungen von Fragen nach dem Klang als Objekt jenseits musikalischer Analysen anregten. ${ }^{14}$ Dass sich die Sound Studies nicht im direkten Anschluss an Rezeptionen poststrukturalistischer Theorien konstituierten, muss man so auch nicht unbedingt als elliptische Gründung durch eine nicht erfolgte Begegnung von musikalischer Nachkriegsavantgarde und French Theory erzählen, wie Steintrager und Chow es suggerieren; man kann ergänzend auf diese Achse der Theoriebildung verweisen, die von Jacques Attali über Michel Serres, Wolfgang Scherer und Kittler bis zu Steve Goodman und Cox verfolgt werden kann. ${ }^{15}$

Dass in Navigating Noise Verhältnisse von Installationskunst und Klimaforschung, White Cube und Weltkriegstechnik zum Teil weniger erklärungsbedürftig scheinen als Zugriffe auf das theoretische Vokabular der Geisteswissenschaften, kann man sowohl als Erbe der Avantgarden als auch als Kontinuität dieser nach wie vor starken antihermeneutischen Diskurstradition sehen. Zur Navigation der komplexen Gemengelage hilfreich erweist sich der Beitrag von Felix Gerloff und Sebastian Schwesinger: "What Does It Mean to Think Sonically? Contours of Noise as a Sonic Figure of Thought» (S.168-190). Der Text situiert den

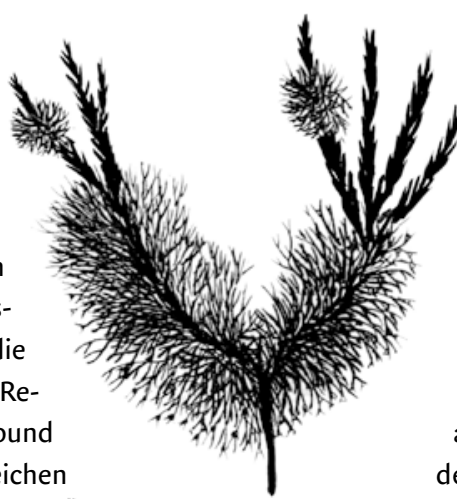
theoretischen Einsatz des Sonischen innerhalb der diskursiven und materiellen Kontaktstellen auditiver Bedeutungsproduktion «between a scientifically objectified physicality of sound and the individual corporealities of hearing and listening" (S.171). Dabei geht es auch um die spezifische Spatialität und Spatiotemporalität von Sound, die in dem Band anhand des titelgebenden Konzeptes der Navigation in den Beiträgen von 


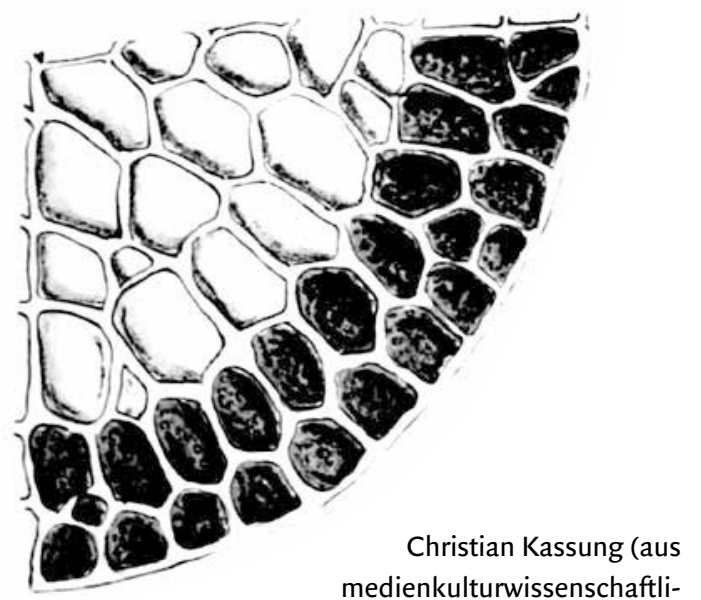

cher Perspektive), Martin Skrydstrup

und Thomas Laepple (aus anthropologischer und klimatologischer Perspektive), Seth Horowitz (aus neuroethologischer Perspektive) und Lino Camprubí (aus wissenschaftshistorischer Perspektive) in ihren sonischen Aspekten entfaltet wird.

In der Vielfalt der Methoden und Gegenstände der drei besprochenen Bände deutet sich eine Zukunft der Sound Studies an, in der mit der zunehmenden Institutionalisierung des Feldes weitere theoretische und fachpolitische Binnendifferenzierungen zu erwarten sind. Diese Entwicklung wird glücklicherweise von fachgeschichtlichen Perspektiven begleitet, die Verhältnisse von Theorie und Gegenständen epistemologisch situieren. Wenn etwas aus der Fachgeschichte der Musikwissenschaft zu lernen ist, dann, dass ontologisierte Gegenstände nicht nur theoretische Invisibilisierungen, sondern auch fachpolitische Probleme erzeugen können. Insbesondere die Medienwissenschaft scheint gegenwärtig geeignet und gefordert, in den Sound Studies Fragestellungen zu entwickeln, die andere Konstellationen als eine Opposition von Repräsentation und Materialität vorsehen. Vielleicht geht das ja sogar ohne Vatermythos.
1 Bettina Schlüter, Axel Volmar: Musikwissenschaft/Sound Studies, in: Jens Schröter (Hg.): Handbuch Medienwissenschaft, Stuttgart 2014, 440-446, hier 445.

2 Vgl. zur Interdisziplinarität der Sound Studies Axel Volmar, Jens Schröter: Einleitung: Auditive Medienkulturen, in: dies. (Hg.): Auditive Medienkulturen. Techniken des Hörens und Praktiken der Klanggestaltung, Bielefeld 2013, 9-34.

3 Innerhalb der Gesellschaft für Medienwissenschaft führten diese Diskussionen im Jahr 2012 zu einer Umbenennung der AG Akustische Medien zur AG Auditive Kultur und Sound Studies, eine Bezeichnung, die nunmehr beide Aspekte versammelt. Vgl. Bettina Schlüter, Axel Vomar: Von akustischen Medien zur Auditiven Kultur. Zum Verhältnis von Medienwissenschaft und Sound Studies, in: dies. (Hg.): Von Akustischen Medien zur Auditiven Kultur. Zum Verhältnis von Medienwissenschaft und Sound Studies (= Navigationen, Bd. 2), Siegen 2015, 7-12, hier 9. Vgl. zum Selbstverständnis der Arbeitsgruppe: gfmedienwissen schaft.de/gesellschaft/ags/ag-auditivekultur-und-sound-studies (9.7.2020).

4 Brian Kane: Sound studies without auditory culture: a critique of the ontological turn, in: Sound Studies, Bd. 1, Nr. 1, 2015, 2-21; Marie Thompson: Whiteness and the Ontological Turn in Sound Studies, in: Parallax, Bd. 23, Nr. 3 , 2017, 266-282; Maren Haffke: Archäologie der Tastatur. Musikalische Medien nach Friedrich Kittler und Wolfgang Scherer, Paderborn 2019. 5 Zu den Unterschieden der Rezeption poststrukturalistischer Theorien in den USA und Deutschland vgl. z. B. Geoffrey Winthrop-Young: Friedrich Kittler zur Einführung, Hamburg 2005, 27-33.

6 Schaeffer als Vertreter der musikalischen Nachkriegsavantgarde repräsentiert eine - wenngleich keineswegs die einzige - wichtige Tradition der Theoriebildung, die in die Formierung der Sound Studies eingeht. Unklar bleibt z. B., warum Steintrager und Chow Schaeffer dem Erfinder der akustischen Ökologie R. Murray Schafer vorziehen, den sie als andere naheliegende Option für einen Gründungsdiskurs nennen (S.14).
7 Vgl. z. B. Christoph Cox: Klangdenken, in: Armen Avanessian u. a. (Hg.): Realismus|Materialismus|Kunst, Berlin 2015, 37-51; Steve Goodman: Sonic Warfare. Sonic Warfare and the Ecology of Fear, Cambridge, London 2012.

8 Auch die Beiträge von Veit Erlmann, Michael Bull, Pooja Rangan, Jairo Moreno und Gavin Steingo, Georgina Born sowie John Mowitt entwickeln ihre Fragestellungen (zu Sound vor Gericht, zu Sirenen, zur Ethnografie, zum Herzschlag, zu Klang als Relation und zum Höhlensound bei Werner Herzog) praktisch ohne direkte Schaeffer-Bezüge.

9 Vgl. Friedrich Kittler: Aufschreibesysteme $1800 \times 1900$, München 1985.

$10 \mathrm{Vgl}$. Wolfgang Ernst: Im Medium erklingt die Zeit. Technische Tempor(e)alitäten und das Sonische als ihre privilegierte Erkenntnisform, Berlin 2015.

$11 \mathrm{Vgl}$. zur Rezeption der materialistischen Medientheorien Jens Gerrit Papenburg: Stop/Start Making Sense, in: Holger Schulze (Hg.): Sound Studies: Traditionen - Methoden - Desiderate. Eine Einführung, Bielefeld 2008, 91-108.

12 Jacques Attali: Noise. The Political Economy of Music, Minneapolis, London 2011. Zum deutschen «Rauschen` vgl. Sabine Sanio, Christian Scheib (Hg.): Das Rauschen. Aufsätze zu einem Themenschwerpunkt im Rahmen des Festivals ‘Musikprotokoll '95 im Steirischen Herbst, Hofheim 1995. Zur ‘Noisiness` von Noise-Konzepten vgl. Marie Thompson: Beyond Unwanted Sound. Noise, Affect and Aesthetic Moralism, New York u. a. 2017.

13 Vgl. Brandon Labelle: Background Noise. Perspectives on Sound Art, New York, London 2006; Douglas Kahn: Earth Sound Earth Signal. Energies and Earth Magnitude in the Arts, Berkeley u. a. 2013.

$14 \mathrm{Vgl}$. Wolfgang Scherer: Babbelogik. Sound und die Auslöschung der buchstäblichen Ordnung, Frankfurt/M. 1983.

15 Vgl. ergänzend zu den schon aufgeführten Bänden Michel Serres: Der Parasit, Frankfurt/M. 1981. 